\title{
OPERATOR SEMISTABLE PROBABILITY \\ MEASURES ON A HILBERT SPACE
}

\author{
R.G. LAHA AND V.K. ROHATG I
}

A characterization of the class of operator semistable probability measures on a real separable Hilbert space is given.

\section{Notation and preliminaries}

Let $H$ be a real separable Hilbert space with inner product $\langle\cdot, \cdot\rangle$ and norm $\|\cdot\|$ and let $B$ denote the $\sigma$-field generated by the class of open subsets of $H$. Let $L=L(H)$ denote the algebra of all bounded linear operators on $H$ with the usual norm topology. For any subset $F$ of $L$, let $\operatorname{Sem}(F)$ denote the closed multiplicative semigroup of operators generated by $F$. Denote by $I$ the identity operator and by 0 , the zero operator. Let $G$ be the multiplicative group of all invertible operators in $L$. Let $P=P(H)$ be the class of all probability measures on $B$.

The convolution of two measures $\mu$ and $\nu$ is denoted by $\mu * \nu$ and the power $\mu^{n}$ is taken in the sense of convolution. Let $\delta_{x}$ denote the probability measure degenerate at $x \in H$. For any $P \in P$ let $\hat{P}$ denote the Fourier transform of $P$. Thus

$$
\hat{P}(y)=\int_{H} \exp (i\langle x, y\rangle) d P(x), y \in H .
$$

Let $\left\{P_{n}\right\}$ be a sequence of probability measures in $P$ and let

Received 5 June 1980. Research supported by NSF Grant MCS78-01338. 
$P \in P$. We write $P_{n} \Rightarrow P$ to say that $\left\{P_{n}\right\}$ converges weakly to $P$. If $P_{n} \Rightarrow P$ we write $P=\lim _{n \rightarrow \infty} P_{n}$.

Let $P \in P$. A closed subset $S(P)$ of $H$ is said to be the support of $P$ if the complement of $S(P)$ has $P$-measure zero and for any $x \in S(P)$ and any neighborhood $V_{x}$ of $x, P V_{x}>0$. We call $P \in P$ fulZ if its support is not contained in any proper hyperplane of $H$. Let $A \in G$, and $P \in P$. Define $A P$ on $B$ by setting

$$
A P(E)=P\left(A^{-1} E\right), \quad E \in B \text {. }
$$

Then $A P \in P$ and

$$
\widehat{A P}(y)=\hat{P}\left(A^{*} y\right), \quad y \in H,
$$

where $A^{*}$ is the adjoint of $A \in L$. Moreover, for $P_{1}, P_{2} \in P$ and $A \in G$ we have

$$
A\left(P_{1} * P_{2}\right)=A P_{1} * A P_{2}
$$

Let $A_{n} \in L, A \in L, P_{n}$ and $P \in P$ such that $A_{n} \rightarrow A$ and $P_{n} \Rightarrow P$. Then $A_{n} P_{n} \Rightarrow A P$.

Given $P \in P$, we define $P^{-} \in P$ by setting $P^{-}(E)=P(-E)$. A measure $P \in P$ is said to be symmetric if $P=P^{-}$. For any $P \in P$, the measure $\hat{P}=P \star P^{-}$is symmetric, and is called the symmetrization of $P$.

In [4] Sharpe introduced and investigated the class of operator stable measures in $\mathbb{R}_{n}$. In particular, he characterized the class of all full operator stable measures. In [2] Kruglov considered the class of semistable measures on $\mathbb{R}_{1}$ and gave a characterization of this class. In [1] Jajte described the class of all full measures on $\mathbb{R}_{n}$ which are operator semistable. In this paper we consider the class of operator-semistable measures on $(H, B)$.

DEFINITION 1. A probability measure $P \in P$ is said to be operator stable, if there exist $A_{n} \in G, x_{n} \in H$, and $Q \in P$ such that the relation 


$$
P=\lim _{n \rightarrow \infty} A_{n^{Q^{n}} * \delta_{n}}
$$

holds.

DEFINITION 2. Let $P \in P$. We say that $P$ is operator semistable if there exist $A_{n} \in G, x_{n} \in H, Q \in P$, and a sequence of positive integers $k_{n}, k_{n} \rightarrow \infty$ as $n \rightarrow \infty$, with $k_{n+1} / k_{n} \rightarrow \gamma$ for some $\gamma \geq 1$ such that

$$
P=\lim _{n \rightarrow \infty} A_{n}{ }^{k}{ }^{n}{ }_{x}
$$

In the following we will assume that the sequence $\left\{A_{n}\right\}, A_{n} \in G$, satisfies the condition: (3) $\operatorname{Sem}\left(\left\{A_{m} A_{n}^{-1}, n=1,2, \ldots, m ; m=1,2, \ldots\right\}\right)$ is compact in the
norm topology of $L$.

We note that for full probability measures on finite-dimensional spaces the compactness condition (3) can be omitted (see, for example, [1]).

We will prove the following theorem which characterizes the class of operator semistable measures on $H$.

THEOREM 1. Let $P$ be a full probability measure on $(H, B)$. Then $P$ is operator semistable if and only if $P$ is infinitely divisible and there exist $a c, 0<c<1$, an element $b \in H$, and an operator $A \in G$, such that the relation

$$
P^{c}=A P \star \delta_{b}
$$

holds. (Here $P^{c}$ is the probability measure corresponding to $[\hat{P}]^{C}$. )

The proof of Theorem $l$ is carried out with the help of several lemmas.

\section{Norming sequences}

We will prove two lemmas concerning the norming sequences $\left\{A_{n}\right\}$ of operators from $G$ satisfying condition (3).

LEMMA 1. Let $P \in P$ be a full operator semistable measure. Then 
$A_{n} \rightarrow 0$ as $n \rightarrow \infty$ where 0 is the zero operator in $H$.

Proof. Let $P \in P$ be a full operator semistable probability measure. Suppose that $A_{n}{ }^{k}{ }^{n} * \delta_{x_{n}} \Rightarrow P$. By symmetrization we see that

$$
\stackrel{\circ}{P}=\lim _{n \rightarrow \infty} A_{n} \stackrel{\circ}{Q}^{k} n
$$

so that

$$
\stackrel{\hat{P}}{P}(y)=\lim _{n \rightarrow \infty}\left[\hat{Q}\left(A_{n}^{*} y\right)\right]^{k}, \quad y \in H
$$

It follows that $\hat{Q}\left(A_{n}^{*} y\right) \rightarrow 1$ as $n \rightarrow \infty$ uniformly in $y \in H$. We show that $A_{n} Q \Rightarrow \delta_{0}$. Let $A$ be an arbitrary limit point of $\left\{A_{n}\right\}$. In view of (3), $\operatorname{Sem}\left(\left\{A_{n}, n \geq 1\right\}\right)$ is compact so that there exists a subsequence $\left\{A_{n_{j}}\right\} \subset\left\{A_{n}\right\}$ such that $A_{n_{j}} \rightarrow A$ as $n_{j} \rightarrow \infty$. It follows that $A_{n_{j}} \stackrel{0}{Q} A Q$ so that $\left\{A_{n} Q\right\}$ is relatively compact and hence tight (Theorem 3.9.2, p. 208 of [3]). From Proposition 7.4 .2 on page 463 of Laha and Rohatgi [3], we conclude that $A_{n} \& \Rightarrow \delta_{0}$.

Let $n \leq n_{j}$. We write

$$
A_{n j} \&^{k_{n}}=A_{n_{j}} 8^{k} * A_{n_{j}} 8^{k_{n}{ }^{-k} n}
$$

Letting $j \rightarrow \infty$ and noting that $A_{n} \& \Rightarrow \delta_{0}$ we conclude from (5) that

$$
\rho=A q^{k} n * P
$$

holds for every $n$.

Next note that $\operatorname{Sem}\left(\left\{A A_{n_{j}}^{-1}, j \geq 1\right\}\right)$ is compact. Let $B$ be a limit point of $\left\{A A_{n_{j}}^{-1}\right\}$. Then without loss of generality, passing to a 
subsequence if necessary we may assume that $A A_{j}^{-1} \rightarrow B$ as $j \rightarrow \infty$.

Consequently,

$$
A=A A_{n_{j} n_{j}}^{-1} \rightarrow B A \text { as } j \rightarrow \infty
$$

so that

$$
A=B A
$$

From (6) we have the equation

$$
\stackrel{\circ}{P}=A A_{n}^{-1} A n_{j} \stackrel{\circ}{Q}^{n_{j}} \stackrel{\circ}{P} .
$$

Letting $j \rightarrow \infty$ we obtain

$$
P=B P \star P
$$

and hence, for $y \in H$,

$$
\hat{P}(y)=\hat{P}\left(B^{*} y\right) \hat{P}(y) \text {. }
$$

Thus

$$
|\hat{P}(y)|^{2}=\left|\hat{P}\left(B^{*} y\right)\right|^{2}|\hat{P}(y)|^{2}, y \in H .
$$

We note that $\hat{P}(y) \neq 0$ in some neighborhood of $0 \in H$. It follows that in some neighborhood of $0 \in H$,

$$
\left|\hat{P}\left(B^{*} y\right)\right|=|\widehat{B P}(y)|=1
$$

and it follows ([3], page 463) that $B P=\delta_{x}$ for some $x \in H$. Since, however, $P$ is a full measure on $B$ this is possible if and only if $B=0$ and $x=0$. From (7) we conclude that $A=0$. Since $A$ is an arbitrary limit point of $\left\{A_{n}\right\}$ we conclude that $A_{n} \rightarrow 0$ as $n \rightarrow \infty$.

LEMMA 2. Let $C$ be a limit point of $\left\{A_{n+1} A_{n}^{-1}, n=1,2, \ldots\right\}$. Then the relation

$$
\hat{P}(y)=[\widehat{C P}(y)]^{\Upsilon} \exp (i\langle b, y\rangle)
$$

holds for all $y \in H$, where $b \in H$ and $\gamma=\lim _{n \rightarrow \infty}\left(k_{n+1} / k_{n}\right) \geq 1$. 
Proof. Let $\left\{A_{n_{j}}\right\} \subset\left\{A_{n}\right\}$ be a subsequence such that $A_{n_{j+1}} A_{j}^{-1} \rightarrow C$ as $j \rightarrow \infty$. Then

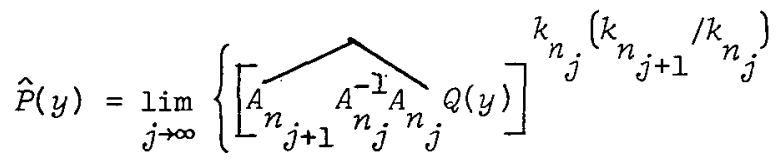

$$
\begin{aligned}
& \cdot \exp \left[i\left(k_{n_{j+1}} / k_{n_{j}}\right)\left\langle A_{n_{j+1}} A_{n}^{-1} x_{n_{j}}, y\right\rangle\right] \\
& \left.\cdot \exp \left[i\left\langle x_{n_{j+1}}-\left(k_{n_{j+1}} / k_{n_{j}}\right) A_{n_{j+1}} A_{n_{j} n_{j}}^{-1}, y\right\rangle\right]\right\} \\
& =\lim _{j \rightarrow \infty}\left\{\left[(\overbrace{A_{j+1} A_{n}^{-1} A_{n} Q(y)})^{k_{n}} \cdot \exp \left(i\left\langle A_{n_{j+1}} A_{n_{j} x_{j}}^{-1}, y\right\rangle\right]\right]^{k_{n} / k_{n}}\right. \\
& \exp \left(i\left\langle b_{n_{j}, y}, y\right)\right\}
\end{aligned}
$$

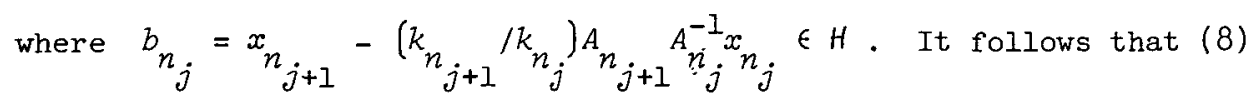
holds for some $b \in H$ where $\left\langle b_{n_{j}}, y\right\rangle \rightarrow\langle b, y\rangle, y \in H$ as $j \rightarrow \infty$.

\section{Proof of Theorem 1}

Suppose that $P$ is infinitely divisible and (4) holds. Set $\gamma=e^{-1}$. Then $\gamma>1$ and we can write (4) as

$$
P=(A P)^{\gamma} * \delta_{\gamma b}
$$

By iteration $n$ times we obtain

$$
P=\left(A^{n} P\right)^{\gamma^{n}} * \delta_{b_{n}}
$$

for some $b_{n} \in H$. Set $k_{n}=\left[\gamma^{n}\right]$. Then $k_{n+1} / k_{n} \rightarrow \gamma$ as $n \rightarrow \infty$. Moreover

$$
P=\lim _{n \rightarrow \infty}\left(A^{n} P\right)^{k}{ }^{n} * \delta_{b_{n}}=\lim _{n \rightarrow \infty} A^{n} P^{k}{ }^{n} * \delta_{b_{n}}
$$


and it follows that $P$ is operator semistable.

Conversely, suppose that $P$ is a full operator semistable probability measure on $B$. Then $P$ is infinitely divisible in view of Lemma 1 . If $\gamma>1$, (4) follows easily from Lemma 2. It remains to prove (4) for the $Y=1$ case. This is done in Lemma 4 below.

LEMMA 3. Let $P \in P$ be a full measure on $B$. Then $P$ is operator stable if and only if for every $n \geq 1$,

$$
P=C_{n} P^{n} * \delta_{n}
$$

where $c_{n} \in G$ and $c_{n} \in H$.

Proof. Clearly if. $P$ satisfies (9) for every $n \geq 1$ then

$$
P=\lim _{n \rightarrow \infty} C_{n} P^{n} * \delta_{n}
$$

so that $P$ is operator stable.

Conversely, suppose that $P$ is a full operator stable measure on $B$. Then there exist $A_{n} \in G, x_{n} \in H$ and $Q \in P$ such that

$$
P=\lim _{n \rightarrow \infty} A_{n} Q^{n} * \delta_{n}
$$

Then, for every $m \geq 1$,

$$
\begin{aligned}
P^{m} & =\lim _{n \rightarrow \infty}\left[A_{n^{Q}}{ }^{n} * \delta_{x_{n}}\right]^{m} \\
& =\lim _{n \rightarrow \infty} A_{n^{Q^{m}} * \delta_{m x_{n}}} .
\end{aligned}
$$

In view of $(10)$

$$
P=\lim _{n \rightarrow \infty} A_{m n^{Q^{m}}} * \delta_{x_{m n}}
$$

Therefore

$$
\begin{aligned}
& P^{m}=\lim _{n \rightarrow \infty} A_{n} Q^{m n} * \delta_{m x_{n}} \\
& =\lim _{n \rightarrow \infty} A_{n m n} A_{m n}^{-1} A^{Q^{m n}} * \hat{o}_{x_{m}} * \delta_{m x}{ }_{n}^{-x_{m}} .
\end{aligned}
$$


In view of condition (3), $\left\{{ }_{A_{n m}}^{A_{m}^{-1}}\right\}$ is compact. Let $C_{m} \in G$ be a limit point of this sequence. Passing to a subsequence if necessary we obtain

$$
P^{m}=C_{m} P \star \delta_{a_{m}}
$$

for some $a_{m} \in H$, and every $m \geq 1$. This completes the proof of Lemma 3 .

LEMMA 4. Let $P \in P$ be fuzz such that

$$
P=\lim _{n \rightarrow \infty} A_{n} Q^{k}{ }^{k} x_{n}
$$

where $A_{n} \in G, x_{n} \in H$ and $k_{n+1} / k_{n} \rightarrow \infty$ as $n \rightarrow \infty$. Then $P$ is operator stable and moreover, the relation (4) holds.

Proof. In view of Lemma $l, P$ is infinitely divisible so that its powers with any positive exponent exist and are infinitely divisible. Let $0<\alpha<1$ be arbitrary. Select, and fix, a sequence $Z(n)$ of integers such that $k_{Z(n)} / k_{n} \rightarrow \alpha$ as $n \rightarrow \infty$. Then, for $y \in H$,

$$
\begin{aligned}
& {[\hat{P}(y)]^{\alpha}=\lim _{n \rightarrow \infty}\left[\widehat{A_{n} Q(y)}\right]^{\alpha k_{n}} \cdot \exp \left(i\left\langle\alpha x_{n}, y\right\rangle\right)} \\
& =\lim _{n \rightarrow \infty}[\overbrace{n^{A} Z(n)^{A} Z(n)^{Q}}^{Q(y)}]^{k_{n}^{\left(k_{Z(n)} / k_{n}\right)}} \cdot \exp \left(i\left\langle\alpha x_{n}, y\right\rangle\right)
\end{aligned}
$$

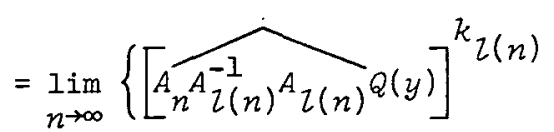

$$
\begin{aligned}
& \text { - } \left.\exp \left\{i\left\langle A_{n} A_{Z(n)}^{-1} x_{Z(n)}, y\right\rangle\right) \cdot \exp \left(i\left\langle\alpha x_{n}-A_{n} A_{Z(n)}^{-1} x_{Z(n)}, y\right\rangle\right)\right\} \\
& =\lim _{n \rightarrow \infty}\left[\widehat{C}_{n^{A} Z(n)}^{Q}(y)\right]^{k} Z(n) \cdot \exp \left(i\left(c_{n} x_{z(n)}, y\right)\right) \cdot \exp \left(i\left(a_{n}, y\right\rangle\right)
\end{aligned}
$$

for some $a_{n} \in H$, where $C_{n}=A_{n} A_{Z(n)}^{-1} \in G$. Write $P_{n}=A_{Z(n)^{Q}}{ }^{k} Z(n) * \delta_{Z(n)}$ and note that $P_{n} \Rightarrow P$ where $P$ is a full probability measure. Also, for $y \in H$, 
(11)

$$
[\hat{P}(y)]^{\alpha}=\lim _{n \rightarrow \infty}\left\{\widehat{C_{n}{ }^{p}}(y) \cdot \exp \left(i\left\langle a_{n}, y\right\rangle\right)\right\}
$$

Note that in view of (3) the sequence $\left\{C_{n}\right\}$ is compact. Let $C_{1 / \alpha} \in G$ be a limit point of $\left\{C_{n}\right\}$ and suppose that $C_{n_{j}} \rightarrow C_{1 / \alpha}$. Then $C_{n_{j}} P_{n_{j}} \Rightarrow C P$ and, moreover,

$$
[\hat{P}(y)]^{\alpha}=\lim _{j \rightarrow \infty}\left\{\widehat{C_{n_{j} n_{j}}}(y) \cdot \exp \left(i\left\langle a_{n_{j}}, y\right\rangle\right)\right\} .
$$

It follows that there exists an element $a_{\alpha} \in H$ such that as $n \rightarrow \infty$,

$$
\left\langle a_{n_{j}}, y\right\rangle \rightarrow\left\langle a_{\alpha}, y\right\rangle, y \in H
$$

Hence

$$
\widehat{C_{n_{j} n_{j}}}(y) \cdot \exp \left(i\left\langle a_{n_{j}}, y\right\rangle\right) \rightarrow \widehat{C_{1 / \alpha}^{P}}(y) \cdot \exp \left(i\left\langle a_{\alpha}, y\right\rangle\right), y \in H,
$$

so that

$$
[\hat{P}(y)]^{\alpha}=\widehat{C_{1 / \alpha}}(y) \cdot \exp \left(i\left\langle a_{\alpha}, y\right\rangle\right)
$$

By uniqueness it follows that the limiting measure is given by

$$
P^{\alpha}=c_{1 / \alpha}{ }^{P * \delta} a_{\alpha}
$$

which is also full. Setting $\alpha=1 / n$ we obtain

$$
P=C_{n} P^{n} * \delta_{n}
$$

for some $c_{n} \in H$ and every $n \geq 1$. It follows at once from Lemma 3 that $P$ is operator stable. Since $0<\alpha<1$ we see from (12) that (4) also holds. This completes the proof of Lemma 4 as well as that of Theorem 1 .

We remark that in view of Lemma 4 the class of operator stable measures on $H$ is a subclass of the class of operator semistable measures on $H$.

Added in proof, 30 October 1980. See the corrigenda, BuzZ. Austral. Math. Soc. 22 (1980), 479-480. 


\section{References}

[1] R. Jajte, "Semi-stable probability measures on $R^{N}$ ", Studia Math. 61 (1977), 29-39.

[2] V.M. Kruglov, "On an extension of the class of stable distributions", Theory Probab. Appl. 17 (1972), 685-694.

[3] R.G. Laha and V.K. Rohatgi, Probability theory (John Wiley \& Sons, New York, Brisbane, Toronto, 1979).

[4] Michael Sharpe, "Operator-stable probability distributions on vector groups", Trans. Amer. Math. Soc. 136 (1969), 51-65.

Department of Mathematics and Statistics,

Bowling Green State University,

Bowling Green,

Oh io 43403 ,

USA.

Present address of first author:

Department of Statistics,

Institute of Advanced Studies,

Australian National University,

PO Box 4,

Canberra,

ACT 2600,

Australia. 